Real Analysis Exchange

Vol. 23(1), 1998-99, pp. 161-174

Jakub Jasinski and Ireneusz Recław, Mathematics Department, University of Scranton, Scranton, PA 18510-2192 e-mail: jsj303@tiger.uofs.edu

\title{
RESTRICTIONS TO CONTINUOUS AND POINTWISE DISCONTINUOUS FUNCTIONS
}

\begin{abstract}
We compare some of the restriction properties that can be found throughout the literature. For example, theorem 10 is a common generalization of three theorems: Blumberg's theorem [2], Baldwin's strengthening of Blumberg's theorem [1], and a related Brown-Prikry's result [8] on Marczewski's $(s)$-measurable functions.
\end{abstract}

\section{Introduction}

In 1922 Blumberg [2] proved that for every function $f: \mathbb{R} \rightarrow \mathbb{R}$ there exists a dense set $X \subseteq \mathbb{R}$, such that $\left.f\right|_{X}$ is continuous. Since then many similar results involving domains and codomains other than $\mathbb{R}$ were obtained. Also many papers can be found, where "continuous" was changed to "differentiable" or "pointwise discontinuous" (i.e., $f: X \rightarrow \mathbb{R}$ is pointwise discontinuous (abbreviated $P W D)$ if $\{x \in X: f$ is continuous at $x\}$ is dense in $X$, see [10] p.105). For a recent comprehensive account of these results see [6]. In this note we would like to compare some restriction properties of real functions defined on separable metric spaces. $\mathbb{R}$ is the set of all real numbers and $\mathbb{Q}$ is the set of rationals. For a set $S$ and a cardinal $\kappa,[S]^{\kappa}=\left\{S^{\prime} \subseteq S:\left|S^{\prime}\right|=\kappa\right\}$. If $\mathcal{F} \subseteq \mathcal{P}(S)$ and $S^{\prime} \subseteq S$, then $\left.\mathcal{F}\right|_{S^{\prime}}=\left\{F \cap S^{\prime}: F \in \mathcal{F}\right\}$. If $\mathcal{F}_{1}, \mathcal{F}_{2} \subseteq \mathcal{P}(S)$, then $\mathcal{F}_{1} \triangle \mathcal{F}_{2}=\left\{F_{1} \triangle F_{2}: F_{i} \in \mathcal{F}_{i}\right.$, for $\left.i=1,2\right\}$. Unless stated otherwise, $X$ will always denote an uncountable, separable metric space, $\mathcal{J}$ will be a proper $\sigma$-ideal on $X$, and $\mathcal{A}$ will be a $\sigma$-algebra of subsets of $X$.

Our goal is to show that given a space $X, \sigma$-algebra $\mathcal{A}$, and a $\sigma$-ideal $\mathcal{J}$ then for every $\mathcal{A}$-measurable function $f: X \rightarrow \mathbb{R}$ there exists a "large"

\footnotetext{
Mathematical Reviews subject classification: Primary: 26A15, Secondary 26A03

Received by the editors January 2, 1997

* Most of this work was completed while the second author was a visiting professor at the University of Scranton.
} 
set $W \subseteq X$ such that the restricted function $\left.f\right|_{W}$ is continuous or pointwise discontinuous. The following six different notions of largeness associated with an ideal $\mathcal{J}$ can be found in restriction theorems stated in [6], [5], [1], [8], [14], and other papers. $W$ is a subset of $X$.

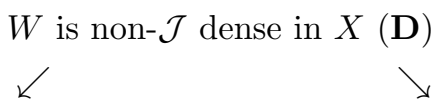

$W$ is non- $\mathcal{J}$ dense in $W(\mathbf{D I}) \quad \operatorname{cl}_{X}(W)$ is non- $\mathcal{J}$ dense in $X(\mathbf{W D})$

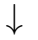

$W \notin \mathcal{J}(\mathbf{N})$

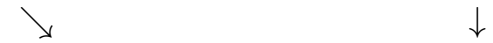

$\operatorname{cl}_{X}(W)$ is non- $\mathcal{J}$ dense in $\operatorname{cl}_{X}(W)($ WDI $)$

$\operatorname{cl}_{X}(W) \notin \mathcal{J}(\mathbf{W N})$

$W$ is non- $\mathcal{J}$ dense in $X$ if $W \cap U \notin \mathcal{J}$ for every nonempty open subset $U \subseteq X$. $\operatorname{cl}_{X}(W)$ stands for the closure of $W$ in $X$. We shall refer to these properties using the bold abbreviations in parenthesis. Here is the key: $\mathbf{D}=$ non- $\mathcal{J}$-Dense, $\mathbf{D I}=$ non- $\mathcal{J}$-Dense in $\mathbf{I}$ tself, $\mathbf{N}=\mathbf{N o t}$ in $\mathcal{J}, \mathbf{W N}=\mathbf{W}$ eakly Not in $\mathcal{J}$ (i.e., not in $\mathcal{J}$ after taking the closure of $W$ ), etc. In general all six are different classes of sets and the above diagram indicates all inclusions.

If $\mathfrak{L}$ is one of those properties (i.e. $\mathbf{D}, \mathbf{D I}, \ldots, \mathbf{W N}$ ), we define a Continuous Restriction Property (C-RP) or a PointWise Discontinuous Restriction Property (PWD-RP) related to $\mathfrak{L}$. Namely, a function $f: X \rightarrow \mathbb{R}$ has a $\mathfrak{L}$-C$\mathrm{RP}$ [resp. $\mathfrak{L}-\mathrm{PWD}-\mathrm{RP}]$ if there exists a set $W \in \mathfrak{L}$ such that $\left.f\right|_{W}$ is continuous [resp. PWD]. We shall say that a pair $(\mathcal{A}, \mathcal{J})$ has a $\mathfrak{L}-\mathrm{C}-\mathrm{RP}$ [resp. $\mathfrak{L}-\mathrm{PWD}-$ $\mathrm{RP}]$ if every $\mathcal{A}$-measurable function $f: X \rightarrow \mathbb{R}$ has the same property. $(\mathcal{A}, \mathcal{J})$ has $\mathcal{A}$-L-C-RP [resp. $\mathcal{A}$ - $\mathfrak{L}$-PWD-RP] if the witness set $W$ can be found in $\mathcal{A}$.

Let $\mathcal{B}(X)$ be the family of all Borel subsets of $X$ and let $\mathcal{B R}(X)$ be the family of all sets with Baire property while $\mathcal{M}(X)$ is the ideal of all subsets of $X$ meager in $X$. So for subsets $X_{1} \subseteq X, \mathcal{M}\left(X_{1}\right)$ is the family of all relatively meager subsets of $X_{1}$. We have $\left.\mathcal{M}\left(X_{1}\right) \subseteq \mathcal{M}(X)\right|_{X_{1}}$. For $X \subseteq \mathbb{R}$ let $\mathcal{L}(X)$ and $\mathcal{N}(X)$ be the Lebesgue measurable and null subsets of $X$. Classic theorems imply that $(\mathcal{B R}(\mathbb{R}), \mathcal{M}(\mathbb{R}))$ has $\mathcal{B R}(\mathbb{R})$-D-C-RP, while the $(\mathcal{L}(\mathbb{R}), \mathcal{N}(\mathbb{R}))$ only has $\mathcal{L}(\mathbb{R})$-DI-C-RP. (See $[8]$ for more details.) 


\section{Continuous Restrictions}

For an arbitrary pair $(\mathcal{A}, \mathcal{J})$ on a separable metric space $X$ we have the following implications.

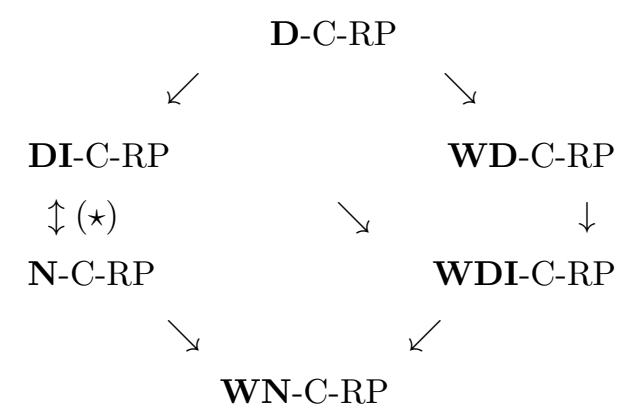

Examples of pairs $(\mathcal{A}, \mathcal{J})$ indicating that, except for $(\star)$, none of these implications may be reversed, can be easily found.

\section{$2.1 \mathcal{A}=\mathcal{P}(X)$}

In $1923 \mathrm{~W}$. Sierpinski and A. Zygmund [17] proved that whenever $|X|=\mathfrak{c}$, then there exists a function $z: X \rightarrow \mathbf{R}$ such that $\left.z\right|_{Y}$ is not continuous for any $Y \in[X]^{\mathfrak{c}}$. This implies that under $\mathrm{CH}(\mathcal{P}(X), \mathcal{J})$ can not have $\mathbf{N}$-C-RP for any $\sigma$-ideal $\mathcal{J}$ containing all singletons. Without $\mathrm{CH}$ however $(\mathcal{P}(\mathbb{R}),[\mathbb{R}] \leq \omega)$ as well as $(\mathcal{P}(\mathbb{R}), \mathcal{M}(X))$ may have $\mathbf{D}$-C-RP. (See [1], [15], and Theorem 2 below.) In ZFC Bradford and Goffman [3] proved that whenever an ideal $\mathcal{J}$ does not contain open sets, then $(\mathcal{P}(X), \mathcal{J})$ has WD-C-RP iff $X$ has no meager open subsets. In general we have the following theorem.

Theorem 1. $(\mathcal{P}(X), \mathcal{J})$ has WDI-C-RP.

Proof. Let $f: X \rightarrow \mathbb{R}$ and suppose that $(\mathcal{P}(X), \mathcal{J})$ does not have the WDIC-RP. By Brown's theorem 2, [5] p.132, we may assume that there exists a subset $X_{1} \subseteq X, X_{1} \notin \mathcal{J}$ such that $\left.\mathcal{M}\left(X_{1}\right) \subseteq \mathcal{J}\right|_{X_{1}}$. Take $X_{1}^{\prime}=X_{1} \backslash \bigcup\{V \subseteq$ $X_{1}: V$ is open in $X_{1}$ and $\left.V \in \mathcal{J}\right\}$. We have $\mathcal{M}\left(X_{1}^{\prime}\right) \subseteq \mathcal{M}\left(X_{1}\right) \subseteq \mathcal{J}$ and the last does not contain open subsets of $X_{1}^{\prime}$. Hence we may apply the above mentioned Bradford-Goffman theorem, [3] p. 667, to $X_{1}^{\prime}$ and obtain a dense subset $W \subseteq X_{1}^{\prime}$, such that $\left.f\right|_{W}$ is continuous. Clearly $\operatorname{cl}_{X}(W) \supseteq X_{1}^{\prime}$ and whenever $U$ is open in $X, U \cap \operatorname{cl}_{X}(W) \neq \emptyset$, then $U \cap X_{1}^{\prime} \notin \mathcal{J}$ by the definition of $X_{1}^{\prime}$.

It is known (see [5], p. 128) that for uncountable separable metric spaces $X$ and any $f: X \rightarrow \mathbb{R}$ there exists a set $W \subseteq X$ such that $\left.f\right|_{W}$ is continuous and 
$|W \cap U| \geq \omega$ for every nonempty open set $U$. Observe that by taking $\mathcal{J}=[X] \leq \omega$ in Theorem 1 above we obtain proposition (C) of [5] and additional property that $\operatorname{cl}_{X}(W)$ is uncountably dense in itself.

If $\mathcal{J}_{1}$ and $\mathcal{J}_{2}$ are ideals on a set $X$ and $Y \subseteq X$, then we say that $\mathcal{J}_{1}$ is orthogonal to $\mathcal{J}_{2}$ on $Y$ if $Y=Y_{1} \cup Y_{2}$ where $Y_{i} \in \mathcal{J}_{i}, i=1,2$. We write " $\mathcal{J}_{1} \perp \mathcal{J}_{2}$ on $Y$ ". Let us consider the following property of a space $X$ and an ideal $\mathcal{J}$ :

$$
X=X_{1} \cup X_{2} \text { where } X_{1} \in \mathcal{M}(X) \text { and } \mathcal{M}\left(X_{2}\right) \subseteq \mathcal{J} \text {. }
$$

It follows from Theorem 1 of [5] that if open subsets of $X$ do not have property $(1)$, then $(\mathcal{P}(X), \mathcal{J})$ has $\mathbf{D}$-PWD-RP. In this context the following theorem is somewhat surprising.

Theorem 2. Suppose that $X$ and $\mathcal{J}$ satisfy (1) and that $\mathcal{J} \backslash \mathcal{M}(X)$ on any open set. Let $f: X \rightarrow \mathbb{R}$ be such that for every Borel set $B \in \mathcal{B}(X) \backslash \mathcal{J}$ the restricted function $\left.f\right|_{B}$ has $N$-C-RP with respect to $\left.\mathcal{J}\right|_{B}$. Then $f$ has $D-C$ - $R P$ with respect to $\mathcal{J}$.

Proof. Let $X=X_{1} \cup X_{2}$ be a partition as in (1). By enlarging $X_{1}$ to a Borel meager set we may assume that $X_{1}, X_{2} \in \mathcal{B}(X)$. Let $\mathcal{U}=\left(U_{n}\right)_{n<\omega}$ be an open basis for $X_{2}$. Non-orthogonality of $\mathcal{J}$ and $\mathcal{M}(X)$ on open sets implies that $U_{n} \notin \mathcal{J}$. Since $U_{n}$ is Borel in $X$, by the N-C-RP of $\left.f\right|_{U_{n}}$ we obtain sets $A_{n} \subseteq U_{n}, A_{n} \notin \mathcal{J}$ such that $\left.f\right|_{A_{n}}$ is continuous. Let $T_{n}=\left\{x \in U_{n}\right.$ : $\exists E_{\text {open }} \subset U_{n}\left(x \in E\right.$ and $\left.\left.A_{n} \cap E \in \mathcal{J}\right)\right\} . X$ is separable so $T_{n} \cap A_{n} \in \mathcal{J}$ and cl $_{U_{n}}\left(T_{n}\right) \backslash T_{n} \in \mathcal{M}\left(X_{2}\right) \subseteq \mathcal{J}$. Furthermore, since $X_{1}$ is meager, $\mathcal{J}$ and $\mathcal{M}(X)$ are non-orthogonal on $U_{n}$. Take $V_{n}=U_{n} \backslash \mathrm{cl}_{U_{n}}\left(T_{n}\right)$ and observe that $C_{n}=A_{n} \cap V_{n}$ is nonempty and non- $\mathcal{J}$ dense in $V_{n}$ for all $n<\omega$.

Now let $W_{n}=C_{n} \backslash \bigcup_{k<n} \operatorname{cl}_{X}\left(V_{k}\right)$ and $W=\bigcup_{n<\omega} W_{n}$. Notice that $W_{n}=$ $\left(V_{n} \cap W\right) \backslash \bigcup_{k<n} \operatorname{cl}_{X}\left(V_{k}\right)$. Hence $W_{n}$ are open in $W .\left.f\right|_{W_{n}}$ is continuous for all $n<\omega$ which implies that $\left.f\right|_{W}$ is also continuous.

To see that $W$ is non- $\mathcal{J}$ dense in $X$ take an arbitrary nonempty open set $T \subseteq X$. Since $X_{2}$ is residual in $X, T_{2}=X_{2} \cap T$ contains some $U_{k}$. Let $k_{0}=\min \left\{k: V_{k} \cap T_{2} \neq \emptyset\right\}$. We clearly have $C_{k_{0}} \cap T_{2} \notin \mathcal{J}$ but also $W_{k_{0}} \cap T_{2} \notin \mathcal{J}$ as all sets of the form $\operatorname{cl}_{X}\left(V_{k}\right) \backslash V_{k}$ are nowhere dense in $X_{2}$ and are in $\mathcal{J}$ by (1). Naturally $W \cap T \notin \mathcal{J}$.

For separable spaces Shelah's theorem 1.4, [15], p. 8, gives the following:

Theorem 3. (Shelah [15]) It is relatively consistent with ZFC that for every function $f: 2^{\omega} \rightarrow 2^{\omega}$ there exists a non-meager subset of $A \subseteq 2^{\omega}$ such that $\left.f\right|_{A}$ is continuous.

Suppose that $X$ is a complete space. Shelah's theorem 3 implies that whenever $B \notin \mathcal{M}(X)$ is a Borel subset of $X$,, then there exists a set $A \in$ 
$\mathcal{P}(B) \backslash \mathcal{M}(B)$ such that $\left.f\right|_{A}$ is continuous. Theorems 3 and 2 yield the following fact.

Corollary 4. It is consistent that for any complete space (or a Borel subset of a complete space without open meager sets) $X$ the pair $(\mathcal{P}(X), \mathcal{M}(X))$ has $D-C-R P$.

Remark 1. It is worth noting that ideals which are ccc in Borel sets have property (1). Suppose that $\mathcal{J}$ is any ccc in Borel sets ideal on $X$ (i.e. $\mathcal{B}(X) \backslash \mathcal{J}$ does not contain uncountable pairwise disjoint subfamilies) and suppose that $\mathcal{M}(X) \nsubseteq \mathcal{J}$. Let $X_{1}^{0} \in \mathcal{M}(X) \backslash \mathcal{J}$ be Borel. For an ordinal $\alpha$ try to find a set $X_{1}^{\alpha} \in \mathcal{B}\left(X \backslash \bigcup_{\beta<\alpha} X_{1}^{\beta}\right) \cap(\mathcal{M}(X) \backslash \mathcal{J})$. By the ccc property this attempt must fail after $\alpha_{0}<\omega_{1}$ steps. Sets $X_{1}=\bigcup_{\alpha<\alpha_{0}} X_{1}^{\alpha}$ and $X_{2}=X \backslash X_{1}$ have the desired properties.

Corollary 4 shows that $\mathrm{CH}$ can not be eliminated from Theorem 1 of [5]. Namely in Shelah's model $(\mathcal{P}(\mathbb{R}), \mathcal{M}(\mathbb{R})$ ) has D-C-RP (in particular it has D-PWD-RP) and $\mathbb{R}$ does not satisfy condition (B') of [5] with property $\mathrm{P}=$ $\mathcal{M}(\mathbb{R})$.

\section{$2.2 \quad \mathcal{A}$-Measurable Functions}

Now we would like to prove a theorem similar to 2 without assuming (1). To compensate for that we are going to work with $\mathcal{A}$-measurable functions and assume $\mathcal{A}$-N-C-RP of $\left.f\right|_{A}$ for all $A \in \mathcal{A} \backslash \mathcal{J}$ i.e., assume that there exists a set $\left.B \in \mathcal{A}\right|_{A} \backslash \mathcal{J}$ such that $\left.f\right|_{B}$ is continuous. Following Bradford and Goffman [3] (see also [13]) we introduce the following definitions: Let $E \subseteq X$, and let $x \in X$. Then $x$ is non- $\mathcal{J}$ relative to $E$ if for every open $V \ni x$ we have $E \cap V \notin \mathcal{J} . x$ is $\mathcal{J}$-heavy relative to $E$ if there exists an open set $U \ni x$ such that all $y \in U$ are non- $\mathcal{J}$ relative to $E$. The first two lemmas are straight forward generalizations of Lemmas 2 and 3 of [3].

Lemma 5. Any subset $E \subseteq X$ can be written as a disjoint union of sets $E=A \cup B_{1} \cup B_{2}$ such that all members of $A$ are $\mathcal{J}$-heavy relative to $E$, $B_{1} \in \mathcal{J}$, and $B_{2}$ is nowhere dense in $X$.

Proof. Let us define $B_{1}=\left\{x \in E: \exists U_{\text {open }} \subseteq X \quad x \in U \&(U \cap E) \in \mathcal{J}\right\}$. $X$ is separable. Hence $B_{1} \in \mathcal{J}$. Now let $B_{2}=\{x \in E: x$ is non- $\mathcal{J}$ but not $\mathcal{J}$-heavy relative to $E\}$. Take an arbitrary open set $T \subseteq X$ and let $x \in B_{2} \cap T$. Since $x$ is not $\mathcal{J}$-heavy, there exists $y \in T$ which is not non- $\mathcal{J}$ relative to $E$. So there exists an open neighborhood $V$ of $y$ such that $E \cap V \in \mathcal{J}$. We must have $V \cap B_{2}=\emptyset$, which shows that $B_{2}$ is nowhere dense. Clearly points of $E$ that are not in $B_{1}$ nor $B_{2}$ are $\mathcal{J}$-heavy. 
For $f: X \rightarrow \mathbb{R}$ we define

$$
H_{f}(X, \mathcal{J})=\left\{x \in X: \forall K_{\text {open }} \ni f(x)\left(x \text { is } \mathcal{J} \text {-heavy relative to } f^{-1}(K)\right)\right\}
$$

Properties of $H_{f}(X, \mathcal{J})$ were studied by Piotrowski [13] in a more general context.

Lemma 6. Let $f: X \rightarrow \mathbb{R}$. There exist sets $B_{1} \in \mathcal{J}$ and $B_{2} \in \mathcal{M}(X)$ such that $H_{f}(X, \mathcal{J})=X \backslash\left(B_{1} \cup B_{2}\right)$.

Proof. Let $\left(G_{n}\right)_{n<\omega}$ be an open basis in $\mathbb{R}$ and let $S_{n}=f^{-1}\left(G_{n}\right)=A^{n} \cup$ $B_{1}^{n} \cup B_{2}^{n}$ where the last union is like in lemma 5. Take $B_{1}=\bigcup_{n<\omega} B_{1}^{n}$ and $B_{2}=\bigcup_{n<\omega} B_{2}^{n}$. Now select an arbitrary $x \in X \backslash\left(B_{1} \cup B_{2}\right)$ and an open set $K \ni f(x)$. Find $n<\omega$ such that $G_{n} \subseteq K$ and $f(x) \in G_{n} . x$ is $\mathcal{J}$-heavy relative to $S_{n}$ so in particular it is $\mathcal{J}$-heavy relative to the bigger set $f^{-1}(K)$. For the other inclusion take $K=G_{n}, n<\omega$ and it follows immediately.

For any ideal $\mathcal{J}$ on a metric space $X$ we define $\mathcal{J}^{*}$ to be the $\sigma$-ideal generated by $\mathcal{J}$ and $\mathcal{M}(X)$. The next lemma is easy to verify.

Lemma 7. Let $Z$ be a separable metric space and let $\mathcal{J}$ be an ideal on $Z$ with $\mathcal{J} \triangle \mathcal{M}(Z)$ on any open set. If $U \subseteq Z$ is open and $V \subseteq U$ is non- $\mathcal{J}^{*}$ dense in $U$, then $\mathcal{J} \perp \mathcal{M}(V)$ on any open subset of $V$.

Lemma 8. Let $Z$ be a zero-dimensional separable metric space. Assume that $\mathcal{J}$ is a $\sigma$-ideal and $\mathcal{A} \supseteq \mathcal{J} \cup \mathcal{B}(Z)$ is a $\sigma$-algebra on $Z$. Suppose that an $\mathcal{A}$ -measurable function $f: Z \rightarrow \mathbb{R}$ and $Y \in \mathcal{A}$ are such that

1) $\mathcal{J} \perp \mathcal{M}(Z)$ on any open subset of $Z$

2) $Y$ is non- $\mathcal{J}$ dense in itself

3) $Y \subseteq H_{f}\left(Z, \mathcal{J}^{*}\right)$

4) $\left.f\right|_{Y}$ is continuous

5) $\left.\left.\forall A \in \mathcal{A} \backslash \mathcal{J} \exists B \in \mathcal{A}\right|_{A} \backslash \mathcal{J} f\right|_{B}$ is continuous.

If $\varepsilon>0$, then there exist pairwise disjoint open subsets $\mathcal{U}=\left(U_{n}\right)_{n<\omega}$ of $Z$ and subsets $Y_{n} \subseteq V_{n} \subseteq U_{n}, Y_{n} \in \mathcal{A}$ such that

6) $\operatorname{diam}\left(U_{n}\right)<\varepsilon$

7) $\cup \mathcal{U}$ is dense in $Z$

8) $V_{n}$ are non- $\mathcal{J}^{*}$ dense in $U_{n}$ 
9) $Y_{n}$ are non- $\mathcal{J}$ dense in itself

10) $\left.f\right|_{Y_{n}}$ is continuous

11) $Y \subseteq \bigcup_{n<\omega} Y_{n}$

12) $Y_{n} \subseteq H_{f}\left(V_{n}, \mathcal{J}^{*}\right)$

13) $\left|f(x)-f\left(x^{\prime}\right)\right|<\varepsilon$ whenever $x, x^{\prime} \in V_{n}$ for some $n<\omega$.

Proof. We shall first define the even numbered sets $U_{n}, V_{n}$, and $Y_{n}$ to satisfy condition 11) and then define the odd numbered ones to satisfy 7$)$. Select $y \in Y$. By 3) there exists an clopen neighborhood $U_{y}^{\prime}$ of $y$ with $\operatorname{diam}\left(U_{y}^{\prime}\right)<\varepsilon$ such that

$$
f^{-1}\left(\left(f(y)-\frac{\varepsilon}{2}, f(y)+\frac{\varepsilon}{2}\right)\right) \text { is non- } \mathcal{J}^{*} \text { dense in } U_{y}^{\prime} .
$$

4) implies the existence of a clopen set $U_{y}^{\prime \prime} \ni y$ such that $\left|f(x)-f\left(x^{\prime}\right)\right|<\frac{\varepsilon}{2}$ whenever $x, x^{\prime} \in Y \cap U_{y}^{\prime \prime}$. Let $U_{y}=U_{y}^{\prime} \cap U_{y}^{\prime \prime}$ and observe that

$$
Y \cap U_{y} \subseteq f^{-1}\left(\left(f(y)-\frac{\varepsilon}{2}, f(y)+\frac{\varepsilon}{2}\right)\right)
$$

Then $\left(U_{y}\right)_{y \in Y}$ is a clopen cover of $Y$. There is a countable set $\left\{y_{n}: n<\omega\right\} \subseteq$ $Y$ such that $\left(U_{y_{n}}\right)_{n<\omega}$ is a subcover of $Y$. Set $G_{n}=U_{y_{n}} \backslash \bigcup_{k<n} U_{k}$. Then $\left(G_{n}\right)_{n<\omega}$ is a disjoint open cover of $Y$ and by possibly deleting some sets we may assure that $G_{n} \cap Y \neq \emptyset$ for all $n<\omega$. For each $n<\omega$ we put $U_{2 n}=G_{n}$, $V_{2 n}=U_{2 n} \cap f^{-1}\left(\left(f\left(y_{2 n}\right)-\frac{\varepsilon}{2}, f\left(y_{2 n}\right)+\frac{\varepsilon}{2}\right)\right)$, and $Y_{2 n}=U_{2 n} \cap Y$.

Assumption 2) implies that $Y_{2 n}$ is non- $\mathcal{J}$ dense in itself. 4) gives continuity of $\left.f\right|_{Y_{2 n}}$. Inclusion (4) shows that

$$
Y_{2 n} \subseteq V_{2 n}
$$

and condition (3) implies that $V_{2 n}$ is non- $\mathcal{J}^{*}$ dense in $U_{2 n}$. Since $\left(G_{n}\right)_{n<\omega}$ was a cover of $Y$, the union $\bigcup_{n<\omega} Y_{2 n}=Y$. 13) follows from the definition of $V_{2 n}$. Hence we have verified all conditions except 7) and 12). While 7) will be taken care of by the odd $U_{n}$-s, 12) for even indices follows from the following claim

Claim: $Y_{2 n} \subseteq H_{f}\left(V_{2 n}, \mathcal{J}^{*}\right)$ for all $n<\omega$.

Let $x \in Y_{2 n}$ and let $K \ni f(x)$ be an open subset of $\mathbb{R}$. Take $K_{1}=K \cap$ $\left(f\left(y_{2 n}\right)-\frac{\varepsilon}{2}, f\left(y_{2 n}\right)+\frac{\varepsilon}{2}\right)$. By $(5) f(x) \in\left(f\left(y_{2 n}\right)-\frac{\varepsilon}{2}, f\left(y_{2 n}\right)+\frac{\varepsilon}{2}\right)$; so $f(x) \in K_{1}$. By the assumption 3$)$ there exists an open subset $U \subseteq Z$ such that $f^{-1}\left(K_{1}\right) \cap U$ is non- $\mathcal{J}^{*}$ dense in $U$. It follows that $f^{-1}\left(K_{1}\right) \cap U \cap G_{n}$ is non- $\mathcal{J}^{*}$ dense in 
$\widehat{U}=U \cap G_{n}$. But since $f^{-1}\left(K_{1}\right) \cap G_{n}$ is a subset of $V_{2 n}$ we obtain that $f^{-1}(K) \cap V_{2 n} \cap \widehat{U}$ is non- $\mathcal{J}^{*}$ dense in $\widehat{U}$. Thus $x \in H_{f}\left(V_{2 n}, \mathcal{J}^{*}\right)$.

To define the odd $U_{n} \supseteq V_{n} \supseteq Y_{n}$ we proceed as in lemma 4 of [3]. Let $R=$ $\left\{z_{\alpha}: \alpha<\kappa\right\}$ for some $\kappa \leq \mathfrak{c}$ be a well-ordering of $H_{f}\left(Z \backslash \mathrm{cl}_{z}\left(\bigcup_{n<\omega} U_{2 n}\right), \mathcal{J}^{*}\right)$. Orthogonality of $\mathcal{J}$ and $\mathcal{M}(Z)$ on every open set and lemma 6 imply that $R$ is dense in $Z \backslash \mathrm{cl}_{z}\left(\bigcup_{n<\omega} U_{2 n}\right)$. Suppose that we have defined sets $U_{\alpha}^{\prime} \supseteq V_{\alpha}^{\prime} \supseteq Y_{\alpha}^{\prime}$ for all $\alpha<\beta<\omega_{1}$. Let $z_{\beta_{0}}$ be the first element of $R \cap Z \backslash \operatorname{cl}_{z}\left(\bigcup_{n<\omega} U_{2 n} \cup\right.$ $\left.\bigcup_{\alpha<\beta} U_{\alpha}^{\prime}\right)$. Let $U_{\beta}^{\prime}$ be a neighborhood of $z_{\beta_{0}}$ disjoint from $\mathrm{cl}_{z}\left(\bigcup_{n<\omega} U_{2 n} \cup\right.$ $\left.\bigcup_{\alpha<\beta} U_{\alpha}^{\prime}\right)$, of diameter less than $\varepsilon$, and such that $V_{\beta}^{\prime}=f^{-1}\left(f\left(z_{\beta_{0}}\right)-\frac{\varepsilon}{2}, f\left(z_{\beta_{0}}\right)+\right.$ $\left.\frac{\varepsilon}{2}\right) \cap U_{\beta}^{\prime}$ is non- $\mathcal{J}^{*}$ dense in $U_{\beta}^{\prime}$. It follows from Lemma 6 that $H_{f}\left(V_{\beta}^{\prime}, \mathcal{J}^{*}\right)$ contains a subset $A \in \mathcal{A} \backslash \mathcal{J}$. By assumption 5), there exists a non- $\mathcal{J}$ dense in itself subset $\left.Y_{\beta}^{\prime} \in \mathcal{A}\right|_{A} \backslash \mathcal{J}$ such that $\left.f\right|_{Y_{\beta}^{\prime}}$ is continuous.

After countably many steps, say $\gamma<\omega_{1}$, the choice of $z_{\gamma_{0}}$ will no longer be possible and this is when $\bigcup_{\alpha<\gamma} U_{\alpha}^{\prime}$ will becomes dense in $Z \backslash \operatorname{cl}_{z}\left(\bigcup_{n<\omega} U_{2 n}\right)$. It suffices to renumerate sets $U_{\alpha}^{\prime}, V_{\alpha}^{\prime}$, and $Y_{\alpha}^{\prime}, \alpha<\gamma$ as $U_{2 n+1}, V_{2 n+1}$, and $Y_{2 n+1}, n<\omega$.

Lemma 9. If $\mathcal{A}$ contains all Borel subsets of $X$ and an $\mathcal{A}$-measurable function $f: X \rightarrow \mathbb{R}$ has $N$-C-RP with respect to $\mathcal{J}$, then it also has $\mathcal{A}-N$ - $C$-RP with respect to the same ideal.

Proof. Suppose that $W \in \mathcal{P}(X) \backslash \mathcal{J}$ is such that $\left.f\right|_{W}$ is continuous. There exists a $G_{\delta}$ subset $G \subseteq X$ and a continuous function $g: G \rightarrow \mathbb{R}$ such that $\left.f\right|_{W} \subseteq g$, see [9]. Since the difference $\left.f\right|_{G}-g$ is also $\mathcal{A}$-measurable, the set $W_{1}=\left(\left.f\right|_{G}-g\right)^{-1}(\{0\})$ is in $\mathcal{A} \backslash \mathcal{J}$. Clearly $\left.f\right|_{W_{1}}$ is continuous.

Theorem 10. Let $X$ be a separable metric space and let $\mathcal{J}$ a $\sigma$-ideal on $X$, $\mathcal{J} \not \mathcal{M}(X)$ on every open set. Suppose that $\mathcal{A} \supseteq \mathcal{J} \cup \mathcal{B}(X)$ is a $\sigma$-algebra on $X$. If $f: X \rightarrow \mathbb{R}$ is $\mathcal{A}$-measurable and $\left.f\right|_{A}$ has $N$-C-RP whenever $A \in \mathcal{A} \backslash \mathcal{J}$, then $f$ has $\mathcal{A}-\boldsymbol{D}$ - $C$ - $R P$.

Proof. Without loss of generality we may assume that $X$ is zero-dimensional. (It suffices to remove a meager set to assure that.) Let $\Delta=\omega^{<\omega} \backslash\{\emptyset\}$. We construct three trees of subsets of $X:\left(U_{\tau}\right)_{\tau \in \Delta},\left(V_{\tau}\right)_{\tau \in \Delta}$, and $\left(Y_{\tau}\right)_{\tau \in \Delta}$.

Claim. There exists an $\mathcal{A}$-measurable non- $\mathcal{J}$ dense in itself subset $Y \subseteq$ $H_{f}\left(X, \mathcal{J}^{*}\right)$ such that $\left.f\right|_{Y}$ is continuous.

$\mathcal{A} \supseteq \mathcal{J} \cup \mathcal{B}(X)$ and Lemma 6 applied to $\mathcal{J}^{*}$ imply that $H_{f}\left(X, \mathcal{J}^{*}\right) \in \mathcal{A} \backslash \mathcal{J}^{*}$. $\left.f\right|_{H_{f}\left(X, \mathcal{J}^{*}\right)}$ has N-C-RP so there exists a set $Y^{\prime} \subseteq H_{f}\left(X, \mathcal{J}^{*}\right), Y^{\prime} \notin \mathcal{J}$ such that $\left.f\right|_{Y^{\prime}}$ is continuous. Since $\mathcal{A} \supseteq \mathcal{B}(X)$, Lemma 9 may be used to extend $Y^{\prime}$ to a subset of $H_{f}\left(X, \mathcal{J}^{*}\right), Y^{\prime \prime} \in \mathcal{A}$ such that $\left.f\right|_{Y^{\prime \prime}}$ is still continuous. Define $Y=Y^{\prime \prime} \backslash\left\{x \in Y^{\prime \prime}: \exists E \subseteq\right.$ open $X\left(x \in E\right.$ and $\left.\left.E \cap Y^{\prime \prime} \in \mathcal{J}\right)\right\} . \quad Y \in \mathcal{A}$ is nonempty because $X$ is separable and non- $\mathcal{J}$ dense in itself. 
To obtain the first level of the three trees apply Lemma 8 with $Z=X, Y=$ $Y$, and $\varepsilon=1$. Now let $k>0$. To obtain sets $\left(U_{\tau^{\wedge} n}\right)_{\tau \in \omega^{k}, n \in \omega},\left(V_{\tau^{\wedge} n}\right)_{\tau \in \omega^{k}, n \in \omega}$, and sets $\left(Y_{\tau^{\wedge} n}\right)_{\tau \in \omega^{k}, n \in \omega}$, from level $k+1$ we apply Lemma 8 for each $\tau \in \omega^{k}$ with $Z=V_{\tau}, Y=Y_{\tau}$ and $\varepsilon=\frac{1}{k+1}$. Then simply put $U_{\tau^{\wedge} n}=U_{n}, V_{\tau^{\wedge} n}=$ $V_{n}$, and $Y_{\tau^{\wedge} n}=Y_{n}$, where $U_{n}, V_{n}$, and $Y_{n}$ are from the Lemma and satisfy conditions 6)-13). Observe that the assumption 1) is preserved from one step to another due to Lemma 7.

Now let $W=\bigcup_{k \in \omega} \bigcup_{\tau \in \omega^{k}} Y_{\tau}$. It is easy to see that for every $k \in \omega$ the union $\bigcup_{\tau \in \omega^{k}} U_{\tau}$ is dense in $X$. To show that $W$ is non- $\mathcal{J}$ dense in $X$ let $T$ be a nonempty open subset on $X$. Due to decreasing diameters of $U_{\tau}$ there exists a $k \in \omega$ and a $\tau \in \omega^{k}$ such that $U_{\tau} \subseteq T$. This implies that $T \cap W \supseteq Y_{\tau} \notin \mathcal{J}$.

It suffices to verify that $\left.f\right|_{W}$ is continuous. Let $x \in W$. For almost all $k \in \omega$ there exist sequences $\tau \in \omega^{k}$ such that $x \in Y_{\tau} . Y_{\tau} \subseteq V_{\tau} \cap W$ and $V_{\tau} \cap W$ is open in $W$ with $\operatorname{diam}\left(f\left(V_{\tau}\right)\right)<\frac{1}{k}$.

The following applications illustrate the strength of theorem 10 .

Corollary 11. (H. Blumberg [2]) If $X$ is a Baire space, then $(\mathcal{P}(X),\{\emptyset\})$ has $D-C-R P$.

Proof. Apply Theorem 10 with $\mathcal{A}=\mathcal{P}(X)$ and $\mathcal{J}=\{\emptyset\}$.

Let $\omega \leq \kappa<\mathfrak{c}$. It is well known (see [16]) that if $X \in[\mathbb{R}]^{\kappa}$ and $f: X \rightarrow \mathbb{R}$, then, under Martin's Axiom, there exists a set $Y \in[X]^{\kappa}$ such that $\left.f\right|_{Y}$ is continuous. Theorem 10 gives the following.

Corollary 12. (S. Baldwin [1]) Assume Martin's Axiom. Let $\omega<\kappa<\mathfrak{c}$, $c f(\kappa)>\omega$. Suppose that $X \subseteq \mathbb{R}$ contains no meager open subsets and $f: X \rightarrow$ $\mathbb{R}$. Then $\left(\mathcal{P}(X),[X]^{<\kappa}\right)$ has $D$-C-RP.

Proof. Clearly, under Martin's Axiom $[X]^{<\kappa} \not \subset \mathcal{M}(X)$ on every open set. Apply theorem 10 with $\mathcal{A}=\mathcal{P}(X)$ and $\mathcal{J}=[X]^{<\kappa}$.

A set $S \subseteq X$ is called ( $s$-measurable if for every perfect set $P \subseteq X$ there exists a perfect subset $P^{\prime} \subseteq P$ such that either $P^{\prime} \cap S=\emptyset$ or $P^{\prime} \subseteq S$. $\left(s_{0}\right)$ is the ideal of hereditarily $(s)$-measurable sets. It is well known (see Marczewski [11]) that whenever $X$ is complete, then $f: X \rightarrow \mathbb{R}$ is $(s)$-measurable iff for every perfect set $P \subseteq X$ there exists a perfect subset $Q \subseteq P$ such that $\left.f\right|_{Q}$ is continuous. It follows that $f$ is $(s)$-measurable iff $\left.f\right|_{A}$ has $(s)$-N-C-RP whenever $A \in(s) \backslash\left(s_{0}\right)$. The following corollary follows from the more general theorem 3 of $[8]$ :

Corollary 13. (Brown and Prikry [8]) If $X$ is a complete space without isolated points, then $\left((s),\left(s_{0}\right)\right)$ has $(s)-D-C-R P$. 
Proof. It is well known that $\left(s_{0}\right) \not \mathcal{M}(X)$ on any open subset of $X$ and that $(s)$ contains all Borel subsets. Theorem 10 completes the proof.

\section{PWD Restrictions}

Now we would like to look at the diagram of pointwise discontinuous restriction properties.

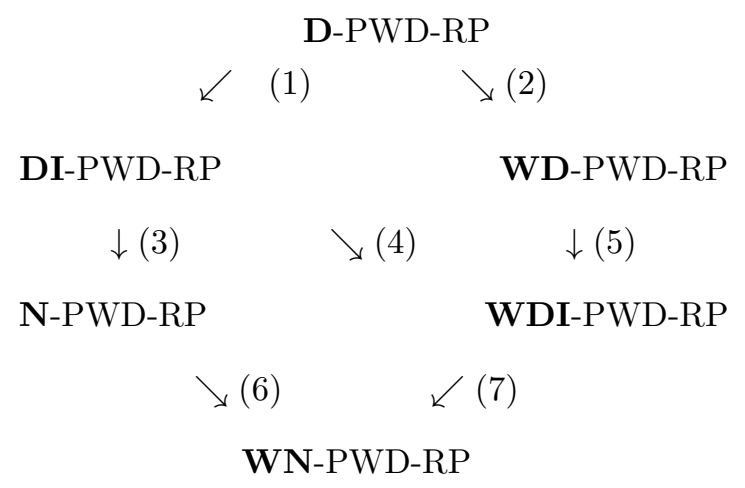

Clearly for any class $\mathfrak{L}$ the $\mathfrak{L}-\mathrm{C}-\mathrm{RP}$ implies the corresponding $\mathfrak{L}-\mathrm{PWD}-\mathrm{RP}$. In addition to that, the following properties are equivalent.

- WD-C-RP $\Longleftrightarrow$ WD-PWD-RP

- WDI-C-RP $\Longleftrightarrow$ WDI-PWD-RP

- WN-C-RP $\Longleftrightarrow \mathrm{WN}-\mathrm{PWD}-\mathrm{RP}$

Hence, we are going to focus on the left side of the diagram. The original Blumberg's theorem [2] implies that $(\mathcal{P}(\mathbb{R}), \mathcal{J})$ has WD-C-RP for any ideal $\mathcal{J}$ without open sets. The following theorem shows that WD-C-RP $\nRightarrow$ N-PWD$\mathrm{RP}\left((4)\right.$ can not be turned by $\left.-90^{\circ}\right)$.

Theorem 14. Let $\mathcal{J}=\left\{M \cup C: M \in \mathcal{M}(\mathbb{R})\right.$ and $\left.C \in[\mathbf{R}]^{<\mathfrak{c}}\right\}$. Then $(\mathcal{P}(\mathbb{R}), \mathcal{J})$ does not have the $N$-PWD-RP.

Proof. Let $z: \mathbb{R} \rightarrow \mathbb{R}$ be the Zygmund-Sierpinski function [17]. Let $A \subseteq \mathbb{R}$ and suppose that $G=\left\{x \in A:\left.z\right|_{A}\right.$ is continuous at $\left.x\right\}$ is dense in $A$. Since $G$ is a relative $G_{\delta}$ subset of $A, A \backslash G \in \mathcal{M}(A) \subseteq \mathcal{J} . z$ is the Zygmund-Sierpinski function; so $G \in[A]^{<\mathfrak{c}} \subseteq \mathcal{J}$. It follows that $A \in \mathcal{J}$. 
Example 15. Implication (1) can not be reversed. Let $K \subseteq \mathbb{R}$ be a nowhere dense perfect set. Take $X=K \cup \mathbb{Q}$ and $\mathcal{J}=[X] \leq \omega . X=\bigcup_{n<\omega} X_{n}$ where $X_{n}$ are pairwise disjoint and nowhere dense in $X$. Assume $X_{1}=K$. Let $f: X \rightarrow \mathbb{R}$, $f(x)=n$ for $x \in X_{n}$. Well known arguments (see [3] p. 667) shows that $(\mathcal{P}(X), \mathcal{J})$ does not have D-PWD-RP. On the other hand $(\mathcal{P}(K),[K] \leq \omega)$ has D-PWD-RP (see [4]); so $(\mathcal{P}(X), \mathcal{J})$ has DI-PWD-RP.

Remark 2. Assume that $\mathcal{J}$ contains all singletons. Under $\mathrm{CH}(\mathcal{P}(X), \mathcal{J})$ has DI-PWD-RP iff it has N-PWD-RP. Suppose that $(\mathcal{P}(X), \mathcal{J})$ does not have DI -PWD-RP. By Brown's theorem 2 of [5] $X=\bigcup_{n<\omega} X_{n}$ where $\mathcal{M}\left(X_{n}\right) \subseteq \mathcal{J}$. Take $z: X \rightarrow \mathbf{R}$ to be the Zygmund-Sierpinski function on $X$. Suppose that $\left.z\right|_{A}$ is pointwise discontinuous for some $A \in \mathcal{P}(X) \backslash \mathcal{J}$. There exists an $n<\omega$ such that $A \cap X_{n} \notin \mathcal{J}$. We can find a set $B, A \supseteq B \supseteq A \cap X_{n}$ such that $\left.z\right|_{B}$ is PWD and $\left|B \backslash\left(A \cap X_{n}\right)\right| \leq \omega$. The set $G=\left\{x \in B:\left.z\right|_{B}\right.$ is continuous at $\left.x\right\}$ is a dense $G_{\delta}$ in $B$. Hence $B \backslash G \in \mathcal{M}(B) \subseteq \mathcal{J}$. Since $B \notin \mathcal{J},|G|>\omega$ which contradicts the Zygmund-Sierpinski property under $\mathrm{CH}$.

\section{Baire, Lebesgue, and Other Measurable Functions}

If $\mathcal{A}$ is the Baire, Lebesgue, universally measurable, or other classic $\sigma$-algebra of sets, then restriction properties for $\left(\mathcal{A}, \mathcal{J}_{\mathcal{A}}\right)$, where $\mathcal{J}_{\mathcal{A}}$ is the ideal of sets hereditarily in $\mathcal{A}$, are discussed in [8]. Here we look at restriction properties for $\mathcal{A}$ with arbitrary ideals $\mathcal{J}$ other than $\mathcal{J}_{\mathcal{A}}$.

If $X$ is meager on itself, then $\mathcal{B R}(X)=\mathcal{P}(X)$ and this case has been discussed above. It follows from a well known theorem of Nikodym [12] that if $X$ does not contain nonempty meager open subsets, then $(\mathcal{B R}(X), \mathcal{M}(X))$ has $\mathcal{B R}(X)$-D-C-RP. Using the same technique we can show that $\left(\mathcal{B R}(X) \triangle \mathcal{J}, \mathcal{J}^{*}\right)$ has $(\mathcal{B R}(X) \triangle \mathcal{J})$-D-C-RP as long as $\mathcal{J} \not \mathcal{M}(X)$ on every open set. It remains to examine pairs $(\mathcal{B R}(X), \mathcal{J})$ where $\mathcal{J} \perp \mathcal{M}(X)$. In such case there exists a nowhere dense set $F \notin \mathcal{J}$. It is easy to find a discrete set $D \subseteq X \backslash F$, such that $\operatorname{cl}_{X}(D) \supseteq F$. This last observation may be applied in a more general situation and yields the following facts.

Proposition 16. If $\mathcal{M}(X) \nsubseteq \mathcal{J}$, then the pair $(\mathcal{P}(X), \mathcal{J})$ has $\mathcal{B R}(X)-N$ $P W D-R P$.

Corollary 17. $(\mathcal{B R}(X), \mathcal{J})$ has $N$-PWD-RP for any $\sigma$-ideal $\mathcal{J}$.

In general N-PWD-RP is the best restriction property that we can hope for $(\mathcal{B R}(X), \mathcal{J})$ where $\mathcal{J} \perp \mathcal{M}(X)$.

Example 18. Let $X=\mathbb{R}=P \cup \dot{\cup}$ where $P$ is some nowhere dense perfect set. Let $\mathcal{M C}(P)=\left\{P^{\prime} \cup P^{\prime \prime}: P^{\prime} \in \mathcal{M}(P)\right.$ and $\left.P^{\prime \prime} \in[P]^{<\mathfrak{c}}\right\}$. Define $\mathcal{J}=\left\{P^{\prime} \cup S^{\prime}\right.$ : 
$P^{\prime} \in \mathcal{M C}(P)$ and $\left.S^{\prime} \subseteq S\right\}$. Clearly $\mathcal{M}(X) \perp \mathcal{J}$ on $X$. $(\mathcal{B R}(X), \mathcal{J})$ does not have DI-PWD-RP because of the following $\mathcal{B R}(X)$-measurable function

$$
f(x)=\left\{\begin{array}{c}
0 \quad \text { if } \quad x \in S \\
z(x) \quad \text { if } \quad x \in P,
\end{array}\right.
$$

where $z$ is the Zygmund-Sierpinski function on $P$. If $W$ was a non- $\mathcal{J}$ dense in itself, then $W \subseteq P$. If $\left.f\right|_{W}$ was PWD, then the set $G=\left\{x \in W:\left.f\right|_{W}\right.$ is continuous at $x\}$ is a dense $G_{\delta}$ subset of $W$ so $W \backslash G \in \mathcal{M}(P)$. This implies that $G \notin \mathcal{J}$ and in particular $|G|=\mathfrak{c}$, but that contradicts the ZygmundSierpinski property.

From Proposition 16 we easily obtain

Corollary 19. If $X$ has positive outer measure, then $(\mathcal{P}(X), \mathcal{N}(X))$ has $N$ $P W D-R P$.

Here also no stronger restriction property is provable due to Example 18. Corollary 19 also follows from Theorem $\mathrm{E}$ of [7] on points of differentiability. More couterexamples for other $\sigma$-algebras follow from the next theorem.

Theorem 20. Let $\mathcal{A}$ be a $\sigma$-algebra of subsets of $\mathbb{R}$ and assume that there exists a set $X \notin \mathcal{M C}=\left\{M \cup C: M \in \mathcal{M}(\mathbb{R})\right.$ and $\left.C \in[\mathbb{R}]^{<\mathfrak{c}}\right\}$ such that $\mathcal{P}(X) \subseteq \mathcal{A}$. If we define $\mathcal{M C}_{X}=\{A \subseteq \mathbb{R}: A \cap X \in \mathcal{M C}\}$, then $\left(\mathcal{A}, \mathcal{M C}_{X}\right)$ does not have the $N-P W D-R P$.

Proof. Follow Example 18 with $P=X$.

Corollary 21. There exists a $\sigma$-ideal $\mathcal{J}$ on $\mathbb{R}$ such that $(\mathcal{L}(\mathbb{R}), \mathcal{J})$ does not have $N$ - $P W D-R P$.

Proof. Use Theorem 20 with $X$ being a second category measure zero set.

Corollary 22. Assume $C H$. If $\mathcal{A}$ is one of the following $\sigma$-algebras: $(s)$ measurable, universally measurable, or $\mathcal{B}(\mathbb{R}) \triangle \mathcal{U N}(\mathbb{R})=\{B \triangle N: B \in \mathcal{B}(X)$ and $N$ is universally null $\}$, then there exists a $\sigma$-ideal $\mathcal{J}$ such that $(\mathcal{A}, \mathcal{J})$ does not have the $N$-PWD-RP.

Proof. Use Theorem 20 with $X$ being a Lusin set.

Remark 3. In the random real model $(\mathcal{B}(\mathbb{R}) \triangle \mathcal{U N}(\mathbb{R}), \mathcal{J})$ has N-PWD-RP for all $\mathcal{J}$. Recall that in this model $\mathcal{U N}(\mathbb{R}) \subseteq[\mathbb{R}] \leq \omega_{1} \subseteq \mathcal{M}(\mathbb{R})$. It follows that $\mathcal{B}(\mathbb{R}) \triangle \mathcal{U N}(\mathbb{R}) \subseteq \mathcal{B R}(\mathbb{R})$ and Corollary 17 implies N-PWD-RP.

The authors are very grateful to Zbyszek Piotrowski for interesting discussions. We also would like to thank Jack Brown. His comments eliminated several errors and clarified some of our proofs. 


\section{References}

[1] S. Baldwin, Martin's axiom implies a stronger version of Blumberg theorem, Real Analysis Exchange 16 (1990/91) 67-73.

[2] H. Blumberg, New properties of all real functions, Trans. Amer. Math. Soc. 24 (1922), 133-128.

[3] J. C. Bradford and C. Goffman, Metric spaces in which Blumberg's theorem holds, Fund. Math. 71 (1974) 243-253.

[4] J. Brown, Metric space in which a strengthened form of Blumberg's theorem holds, Fund. Math. 71 (1971) 243-253.

[5] J. Brown, Variations on Blumberg theorem, Real Analysis Exchange 9 (1983/84) 123-137.

[6] J. Brown, Restriction theorems in real analysis, Real Analysis Exchange $20(1994 / 95)$ 510-526.

[7] J. Brown, A measure theoretic variant of Blumberg's Theorem, Proc. Amer. Math. Soc. 66 (1977), p. 266-268.

[8] J. Brown and K. Prikry, Variations on Lusin's theorem, Trans. Amer. Math. Soc. 302 (1987) 77-86.

[9] R. Engelnking, General Topology, Helderman-Verlag, Berlin 1989.

[10] K. Kuratowski, Topology, vol. I, Academic Press, New York 1966.

[11] E. Marczewski, Sur un classe de fonctions de W. Sierpinski et la classe correspondante, Fund. Math. 24 (1935) 17-34.

[12] O. Nikodym, Sur la condition de Baire, Bull. Acad. Pol. (1929) 591.

[13] Z. Piotrowski, Ideal Banach Category Theorems and Functions, Math. Bohemica (to appear).

[14] I. Recław, Restrictions to continuous functions and Boolean algebras, Proc. Amer. Math. Soc. 118 no 3 (1993) 791-796.

[15] S. Shelah, Possibly every real function is continuous on a non-meager set, Publ. Inst. Math. (Beograd) (N.S.) 57 (71) (1995), 47-60.

[16] J. Shinoda, Some consequences of Martin's Axiom and negation of the Continuum Hypothesis, Nogoya Math. J. 49 (1973) 117-125. 
[17] W. Sierpinski and A. Zygmund, Sur une fonction qui est discontinue sur tout ensemble de puissance du continu, Fund. Math. 4 (1923) 316-318. 